\title{
COMMENTARY
}

\section{Inspiratory muscle training in difficult to wean patients: work it harder, make it better, do it faster, makes us stronger}

\author{
Stefano Nava* and Luca Fasano \\ See related research by Martin et al., http://ccforum.com/content/15/2/R84
}

\begin{abstract}
Weaning from prolonged mechanical ventilation is a complex, time-consuming process that involves the loss of force/generating capacity of the inspiratory muscle. In their study'Inspiratory muscle strength training improves the outcome in failure to wean patients: a randomized trial', Martin and colleagues showed that the use of an inspiratory muscle strength program increased the maximal inspiratory pressure and improved weaning success compared to a control group. The study was performed mainly in postsurgical patients, however, and the results, therefore, may not be generalizable to other subsets of patients, such as those with chronic obstructive pulmonary disease or congestive heart failure. Indeed, the study applied so-called 'strength training' and not 'endurance training', which may be more appropriate in certain circumstances.
\end{abstract}

Martin and colleagues [1] recently published in Critical Care an article entitled 'Inspiratory muscle strength training improves the outcome in failure to wean patients: a randomized trial'. Failure-to-wean patients comprise a subset of patients receiving mechanical ventilation who have weaning difficulties such that the duration of ventilation may be abnormally prolonged ( $>15$ days). In the US, about 300,000 patients every year receive prolonged life support in the ICU, and this number is likely to double within a decade, with associated costs of more than US\$ 50 billion [2]. Weaning from prolonged mechanical ventilation is a complex, time-consuming process that does not involve just selecting the best ventilation method for a particular patient.

*Correspondence: stefano.nava@aosp.bo.it

Respiratory and Critical Care Unit, Sant'Orsola Malpighi Hospital, 40138 Bologna, Italy
Due to the nature of critical illness and the modalities used to manage it, prolonged bed rest, with well-known adverse physiological effects, seems to be the rule in the ICU. Rehabilitation has the potential to restore lost function, but with few exceptions is traditionally not started until after ICU discharge since critically ill patients are often viewed as 'too sick' to tolerate a retraining program. Several reports have shown that the diaphragm and the other respiratory muscles are weak in these patients; this occurs for many reasons, including the effects of controlled mechanical ventilation (that is, disuse atrophy), electrolyte depletion, use of drugs affecting inotropic properties (that is, steroids and myorelaxants), and the presence of co-morbidities (for example, chronic obstructive pulmonary disease (COPD), congestive heart failure and sepsis) [3]. Despite the fact that respiratory muscle fatigue has not been demonstrated so far, even in patients sufficiently ill to require mechanical ventilation [4], the loss of force/ generating capacity (that is, the reduction in maximal inspiratory pressure) seems to be a major determinant of weaning failure, although it is not a specific predictor of inability to sustain totally unsupported breathing, but one of a few physiological parameters that improve in those patients finally liberated from the ventilator after several unsuccessful attempts [5].

There is therefore a theoretical rationale for starting, as soon as clinical conditions are stabilized, a specific inspiratory muscle training (IMT) program in patients with weaning difficulties in order to make them work harder, so they can get stronger. The use of IMT has been shown to have beneficial effects in spontaneously breathing patients undergoing massive surgical procedures [6], although despite several studies, the effectiveness of IMT in COPD is still controversial [7]. Very few and only uncontrolled studies have been performed in mechanically ventilated patients [8,9], so the first published randomized controlled trial, Martin and colleagues [1], is very welcome.

Martin and colleagues have shown in their elegant study performed on difficult to wean patients that the use 
of an IMT program can lead to increased maximal inspiratory pressure and improved weaning success compared to the control group. The merits of the study are numerous, including the rigorous methodology, the use of a 'real' control (the SHAM group), the careful selection of patients and the recording and analysis of many confounding variables (the use of drugs during the hospital stay and all complications), but we would also like to highlight some questions that this investigation leaves unanswered.

\section{Is IMT useful in all patients subjected to prolonged mechanical ventilation?}

In their study, Martin and colleagues [1] enrolled mainly post-surgical patients, representing only a minority of all ventilated patients in 'real world' ICUs. Indeed, and most importantly, the majority of patients having weaning difficulties are those affected by chronic respiratory disorders (COPD), and in these individuals the use of IMT may be theoretically useless or even detrimental. Levine and colleagues [10] have shown, for example, that the muscle fibers of the diaphragm in COPD patients are adapted in such a way that they increase resistance to fatigue, so that any form of training may not be able to improve their already satisfactory endurance. Indeed, as pointed out by Martin and colleagues [1] in the Discussion section of their article, the possibility of exercise-induced muscle damage during IMT should be considered, particularly in those patients where the severity of airflow obstruction is associated with diaphragm injury even in a phase of clinical stability [11].

\section{What is the best IMT schedule for these patients?}

The duration of muscle loading during each IMT session in the study by Martin and colleagues [1] was 1 minute per day at an intensity of about $30 \%$ of the maximal inspiratory pressure generated at the beginning of the trial. This schedule may be considered as 'strength training' (that is, high intensity and a low number of repetitions), while 'endurance training' consists of low intensity and a high number of repetitions. Intuitively, one may think that the resistance to fatigue in difficult to wean patients may be more important than increasing the force generation itself (consider the concept of a sprinter versus a marathon runner), but this needs to be addressed in the future, in both clinical studies comparing the two training modalities and physiological studies.

\section{Is the higher weaning success due to IMT?}

The study by Martin and colleagues [1] simply showed that those patients undergoing IMT had a higher percentage of weaning success. As a matter of fact, comparable improvements in maximal diaphragm pressure were obtained in successfully weaned patients, compared to the first unsuccessful weaning trial, in a very similar group of patients who had simply undergone a rehabilitation program without specific IMT [5].

\section{How could the results of this study influence our daily practice?}

The IMT, as proposed by Martin and colleagues [1], is rather simple to perform, but in our view some recommendations do need to be given. Firstly, the protocol should be applied to patients with documented inspiratory muscle weakness, which was surprisingly not considered in the inclusion criteria. Secondly, the measurement of maximal inspiratory pressure is volitional and great attention should therefore be paid to how to perform the maneuver. We suggest to use the procedure proposed by Vitacca and colleagues [12], who studied different methods to record maximal inspiratory pressure in spontaneously breathing patients with tracheotomy, like those enrolled in the study by Martin and colleagues [1]. The IMT proposed in the study implies that the patients were able to achieve spontaneous breathing autonomy and to breathe without supplemental oxygen, largely reducing the application of the protocol [1].

\section{Conclusion}

IMT may help difficult to wean patients to 'work it harder', but further studies are certainly needed to assess if it may work to 'do it [the weaning process] faster'.

\section{Abbreviations}

COPD, chronic obstructive pulmonary disease; IMT, inspiratory muscle training.

Competing interests

The authors declare that they have no competing interests.

\section{Acknowledgements}

The phrase 'work it harder, make it better, do it faster, makes us stronger' is from Harder, Better, Faster, Stronger, an album by Daft Punk Discovery and produced by Virgin Records October 2001.

\section{Published: 18 April 2011}

\section{References}

1. Martin DA, Smith BK, Davenport P, Harman E, Gonzalez-Rothi RJ, Baz M, Layon J, Banner M, Caruso L, Deoghare H, Tseng-Tien Huang, Gabrielli A: Inspiratory muscles strength training improves the outcome in failure to wean patients: a randomized trial. Crit Care 2011, 15:R84.

2. Zilberberg MD, Shorr AF: Prolonged acute mechanical ventilation and hospital bed utilization in 2020 in the United States: implications for budgets, plant and personnel planning. BMC Health Serv Res 2008, 8:242.

3. Tobin MJ, Laghi F, Brochard L: Role of respiratory muscles in acute respiratory failure of COPD: lessons from weaning failure. J App/ Physiol 2009,107:962-970.

4. Laghi F, Cattapan SE, Jubran A, Parthasarathy S, Warshawsky P, Choi YS, Tobin $\mathrm{MJ}$ : Is weaning failure caused by low-frequency fatigue of the diaphragm? Am J Respir Crit Care Med 2003, 167:120-127.

5. Carlucci A, Ceriana P, Prinianakis G, Fanfulla F, Colombo R, Nava S: Determinants of weaning success in patients with prolonged mechanical ventilation. Crit Care 2009, 13:R97.

6. Hulzebos EH, Helders PJ, Favie NJ, De Bie RA, Brutel de la Riviere A, Van Meeteren NL: Preoperative intensive inspiratory muscle training to prevent 
postoperative pulmonary complications in high-risk patients undergoing cabg surgery: a randomized clinical trial. JAMA 2006, 296:1851-1857.

7. Gosselink R, De Vos J, van den Heuvel SP, Segers J, Decramer M, Kwakkel G: Impact of inspiratory muscle training in patients with COPD: what is the evidence? Eur Respir J 2011, 37:416-425.

8. Martin AD, Davenport PD, Franceschi AC, Harman E: Use of inspiratory muscle strength training to facilitate ventilator weaning: a series of 10 consecutive patients. Chest 2002, 122:192-196.

9. Sprague SS, Hopkins PD: Use of inspiratory strength training to wean six patients who were ventilator-dependent. Phys Ther 2003, 83:171-181.

10. Levine S, Kaiser L, Leferovich J, Tikunov B: Cellular adaptations in the diaphragm in chronic obstructive pulmonary disease. N Eng/ J Med 1997, 337:1799-1806
11. MacGowan NA, Evans KG, Road JD, Reid WD: Diaphragm injury in individuals with airflow obstruction. Am J Respir Crit Care Med 2001, 163:1654-1659.

12. Vitacca M, Paneroni M, Bianchi L, Clini E, Vianello A, Ceriana P, Barbano L, Balbi $B$, Nava S: Maximal inspiratory and expiratory pressure measurement in tracheotomised patients. Eur Respir J 2006, 27:343-349.

doi:10.1186/cc10125

Cite this article as: Nava S, Fasano L: Inspiratory muscle training in difficult to wean patients: work it harder, make it better, do it faster, makes us stronger. Critical Care 2011, 15:153. 